\title{
Perforating branches of the internal thoracic artery in women with breast cancer: An anatomical study for breast-conservation surgery
}

\author{
KIMIKO NAKATANI ${ }^{1}$, HIRONORI MAEDA ${ }^{2}$, YOUSUKE TANAKA ${ }^{2}$, NORIHIKO HAMADA ${ }^{1}$, \\ AKIHITO NISHIOKA $^{1}$, SHIRO SASAGURI ${ }^{2}$ and YASUHIRO OGAWA ${ }^{1}$
}

Departments of ${ }^{1}$ Diagnostic Radiology and Radiation Oncology, ${ }^{2}$ Surgery, Medical School, Kochi University, Oko-cho, Kohasu, Nankoku city, Kochi 783-8505, Japan

Received October 17, 2007; Accepted January 29, 2008

\begin{abstract}
Breast reconstruction after breast-conservation surgery is an important issue for breast cancer patients. Various factors are associated with complications, of which blood flow is one of the more important. The perforating branches of the internal thoracic artery (ITA) are key contributors to blood flow in the anterior chest wall. The present study examined the distributions and depths of the perforating branches of the internal thoracic artery using a multi-detector row-computed tomography (MDCT) angiography. The subjects in this prospective study comprised of 38 women with suspected breast cancer who underwent MDCT angiography. The images were analyzed on computer using transverse MDCT source data and volume renderings. A total of 47 perforating branches were found, with $27(57.4 \%)$ originating in the second, 6 $(12.8 \%)$ in the third and fourth, $5(10.6 \%)$ in the first and 2 branches $(4.3 \%)$ originating in the fifth intercostal space, with one branch $(2.1 \%)$ originating opposite the first intercostal space. A strong correlation was identified between the distance from the skin to the branch and adipose thickness at the shallowest and deepest points $(\mathrm{P}<0.001)$. The distributions and depths of the perforating branches of ITA identified in this study may be helpful in immediate breast reconstruction following mastectomy or breast-conserving surgery.
\end{abstract}

\section{Introduction}

Immediate breast reconstruction following mastectomy or breast-conserving surgery has been shown to exert a beneficial effect on the overall psychological well-being of women receiving treatment for breast cancer $(1,2)$. Many surgical

Correspondence to: Dr Kimiko Nakatani, Department of Diagnostic Radiology and Radiation Oncology, Medical School, Kochi University, Oko-cho, Kohasu, Nankoku city, Kochi 783-8505, Japan E-mail: jm-nakakimi@kochi-u.ac.jp

Key words: anatomy, breast cancer, multi-detector row-computed tomography angiography, perforating branch of internal thoracic artery techniques have been described, such as reconstruction using a transverse rectus abdominus myocutaneous (TRAM) flap following mastectomy, or a latissimus dorsi muscle flap immediately after breast-conserving surgery (3-10). Although blood flow in a pedicle flap such as the latissimus dorsi muscle flap is usually maintained, reconstructed breast scleroses and adiponecrosis occasionally appear long after breastconservation treatment $(5,11,12)$. Of course, adjuvant chemotherapy and/or radiotherapy in breast conservation treatments may influence these complications.

Major arterial inflow to the mammary glandular tissue comes from the perforating branches of the internal thoracic (ITA), lateral thoracic and thoracodorsal artery. Breast reconstruction with consideration for the breast blood supply, such as perforating branches of the ITA, offers the potential to avoid such complications. Breast reconstruction with the preservation of a perforating artery is reportedly associated with fewer complications $(6,13)$. The existing literature regarding ITAperforating branches has focused on the beginning of such branches for microsurgical breast reconstruction. However, not all institutions can perform and not all breast cancer patients require microsurgery. To the best of our knowledge, no reports have described ITA-perforating branches in terms of origin and distribution.

Digital subtraction angiography (DSA) is currently the imaging technique of choice for assessing peripheral arteries. In recent years, multi-detector row-computed tomography (MDCT) angiography has shown great potential for the minimally invasive assessment of peripheral arteries (14-16).

By using MDCT angiography, we confirmed that perforating branches of the ITA run to the surface of the thoracic cage through intercostal spaces, and then often display a tortuous run through thoracic adipose tissue. We have encountered wide interindividual variations in the courses and lengths of these perforating branches. The aim of the present study was to determine the locations, courses and distributions of perforating branches of the ITA using MDCT angiography.

\section{Materials and methods}

Subjects comprised of 38 women (mean age, 56.4 years; range, 38-82) with breast cancer who underwent chest MDCT 


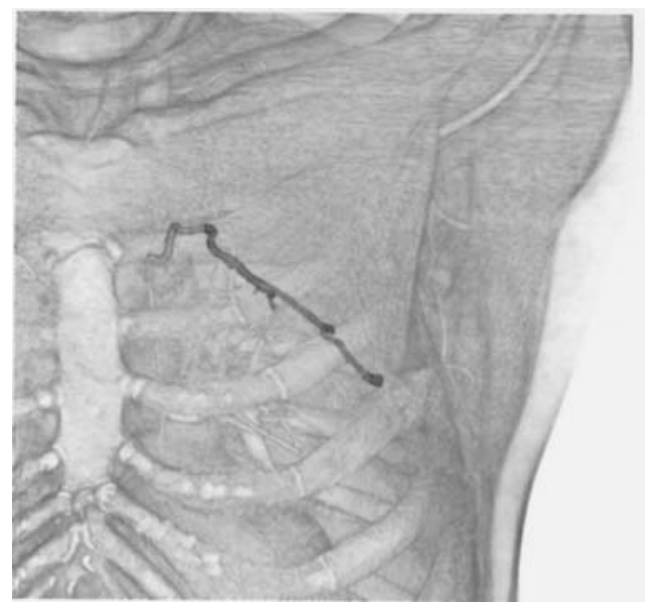

Figure 1. Three-dimensional images based on MDCT using volumerendering techniques. The identified perforating branches were clearly displayed with the precordial muscle and bone. This perforating artery originates in the second intercostal space and runs left-caudally.

between April 2004 and December 2005 at Kochi Medical School Hospital, Japan. All study protocols were approved by the institutional review board of Kochi Medical School, Japan, and written informed consent was obtained from all patients prior to enrolment in this prospective study. All of the patients underwent operation. Surgical staging (TNM 2004 classification) was identified as follows: stage 0 (carcinoma in situ), $\mathrm{n}=1$; stage $1, \mathrm{n}=12$; stage $2 \mathrm{~A}, \mathrm{n}=11$; stage $2 \mathrm{~B}, \mathrm{n}=7$; stage $3 A, n=6$ and stage $3 B, n=1$. Breast cancer affected the left side in 18 women and the right side in 20 women.

MDCT angiography was performed using an Aquilion 16-detector row CT scanner (Toshiba Medical Systems, Tochigi, Japan). The total volume of iopamidol as contrast material (Iopamiron 370, Schering, Osaka, Japan) was dependent on the body weight of the patient $(1.2 \mathrm{ml} / \mathrm{kg})$. Contrast material was administered using a dual shot automated injector (Nemoto Kyorindo, Tokyo, Japan) at a flow rate of $0.06 \mathrm{ml} / \mathrm{sec} / \mathrm{kg}$ through a 22 -gauge needle placed in an antecubital or basilic vein of the unaffected side. At the same flow rate, $50 \mathrm{ml}$ of saline was intravenously injected after contrast material administration. Continuous low-dose monitoring examinations $(120 \mathrm{kV}, 50 \mathrm{mAs}, 0.5$-sec scan time) were performed $5 \mathrm{sec}$ after the start of the contrast material injection. At $5 \mathrm{sec}$ after the preset contrast enhancement level reached 100 Hounsfield units (HU) in the region of interest placed in the aortic arch, CT was automatically initiated $(120 \mathrm{kV}$, automatically changed voltage, $0.5-\mathrm{sec}$ scan time). Data acquisition was performed in a craniocaudal direction with a nominal section thickness of $1.0 \mathrm{~mm}$, table feed of $30 \mathrm{~mm} / \mathrm{sec}$ and a 0.5 -sec gantry rotation time (beam pitch, 0.94). The reconstructed field of view displayed a mean diameter of $235 \mathrm{~mm}$ (range, 179-281), resulting in a mean pixel size of $0.46 \mathrm{~mm}$ (range, $0.35-0.55$ ).

The MDCT data were transferred to a dedicated workstation (Virtual Place Advance PLUS, AZE, Tokyo, Japan) to measure the distance from the skin to the artery and create volume-rendered images of the ITA-perforating arteries of the precordia. The images were analyzed on the basis of transverse CT source data and volume renderings

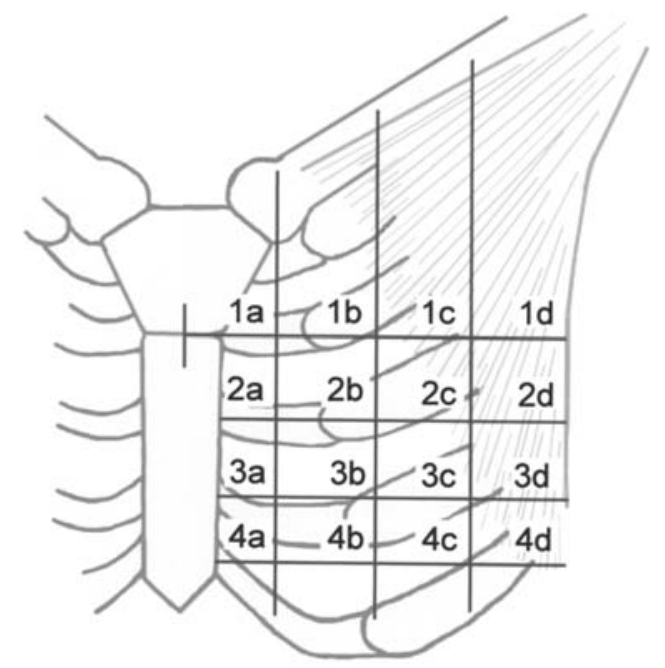

Figure 2. Partitioning of the precordial region. The horizontal lines pass through the sternal angle and third, fourth and fifth sternocostal joints. The vertical lines quadrisect from the medial sternal line to the lateral margin of the pectoralis major muscle.

Table I. ITA-perforating branches.

\begin{tabular}{lccr}
\hline & \multicolumn{3}{c}{ Number (\%) } \\
\cline { 2 - 4 } Intercostal space & Right & Left & Total \\
\hline 1st & $2(7.7)$ & $3(14.3)$ & $5(10.4)$ \\
2nd & $14(53.8)$ & $13(61.9)$ & $27(56.3)$ \\
3rd & $3(11.5)$ & $3(14.3)$ & $6(14.6)$ \\
4th & $5(19.2)$ & $1(4.8)$ & $6(12.5)$ \\
5th & $1(3.8)$ & $1(4.8)$ & $2(4.2)$ \\
Opposite 1st & $1(3.8)$ & $0(0.0)$ & $1(2.1)$ \\
Total & 26 & 21 & 47 \\
\hline
\end{tabular}

were available at the workstation. All measurements and reconstructions were performed by the same radiologist (N.K.) who had 5 years experience in reading MDCT and 3 years experience in three-dimensional post-processing techniques.

The perforating branches of the affected ITA were identified at the workstation using transverse sections and three-dimensional images. The identified perforating branches were displayed with muscles of the precordia using volumerendering techniques (Fig. 1). The precordial region was partitioned by using 4 horizontal lines passing through the sternal angle and third, fourth and fifth costal cartilages, and 3 vertical lines spaced from the midline of the sternum to the lateral margin of the pectoralis major muscle. The areas divided by these lines were numbered as shown in Fig. 2. The distances from the skin to the branch (b) and adipose thickness (a) at all curved points were measured and the b/a ratio was calculated. The point of the lowest b/a ratio was defined as point $S$, and the highest as point $D$. Distances from the midline to points $\mathrm{S}$ and $\mathrm{D}$ were measured and defined as distances $\mathrm{S}$ 
Table II. A combination of double ITA-perforating branches.

\begin{tabular}{lc}
\hline Intercostal space & $\mathrm{n}$ \\
\hline 1st and 2nd & 1 \\
1st and 4th & 2 \\
2nd and 3rd & 3 \\
2nd and 4th & 3 \\
2nd and 5th & 1 \\
Opposite 1st and 5th & 1 \\
Total & 11 \\
\hline
\end{tabular}

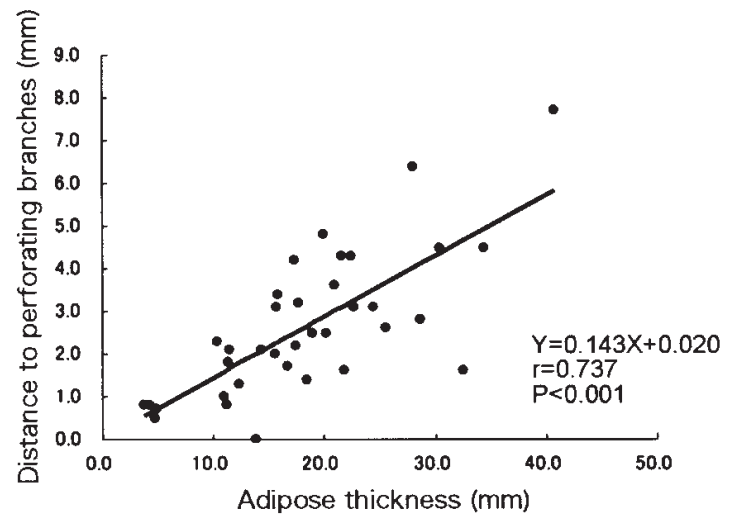

Figure 3. The relationship between the distance from the skin to perforating branches and adipose thickness at a point where branches run most shallowly. The solid line indicates a regression line.

and $\mathrm{D}$, respectively. The distance from the midline to the nipple was defined as distance $\mathrm{N}$. The authors performed these measurements and calculations as baseline data for surgeons resecting breast cancer with consideration for the perforating branches of the ITA.

\section{Results}

The numbers of perforating branches that were detected in this CT study were 1 in 25 cases $(65.8 \%)$ and 2 in 11 cases (28.9\%). In 2 cases $(5.3 \%)$, although thought to be present, no perforating artery was detected in this study because a DSA study was not performed. A total of 47 perforating branches were found.

The origin of ITA-perforating branches. Of the 47 perforating branches, $27(57.4 \%)$ originated in the second intercostal space and $6(12.8 \%)$ in the third and fourth intercostal spaces (Table I). For the 11 cases displaying the 2 perforating branches, the most common origins were the second and third or second and fourth intercostal spaces (3 cases each, 27.3\%). Table II shows detailed results.

The distribution of ITA-perforating branches. Distributions were checked for a total of 47 branches in 36 cases. Of the 5 branches originating from the first intercostal space, all five

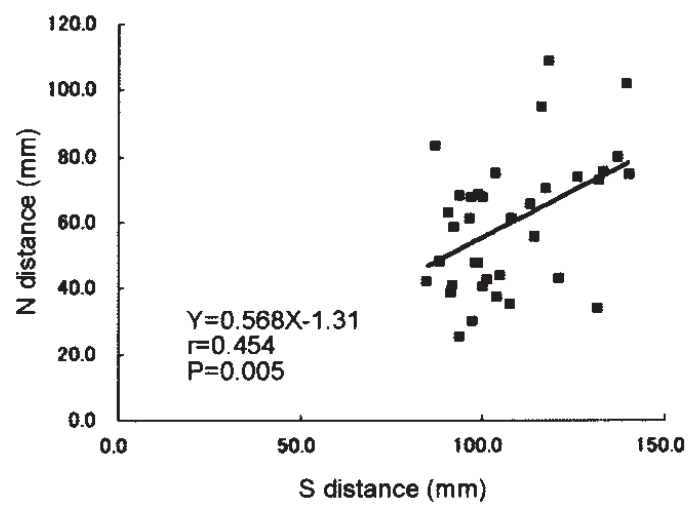

Figure 4. The relationship between the distance from the midline to the point at which branches run most shallowly ( $\mathrm{S}$ distance) and from the midline to the nipple ( $\mathrm{N}$ distance). The solid line indicates a regression line.

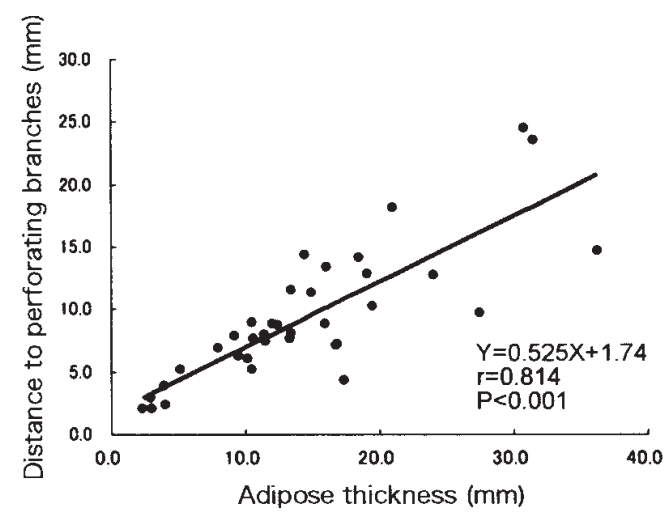

Figure 5. The relationship between the distance from the skin to the perforating branches and adipose thickness at the point where the branches run most deeply. The solid line indicates a regression line.

$(100 \%)$ ran through area $1 \mathrm{a}$, while $4(80 \%)$ ran through area $1 \mathrm{~b}$. Of the 27 branches from the second intercostal space, 21 $(77.8 \%)$ ran through area $2 \mathrm{a}$. Subsequently, 7 out of 21 branches running through $2 \mathrm{a}$ ran through area $1 \mathrm{~b}$ from area 1a, 4 ran through area $1 \mathrm{~b}$ directly and 9 ran through area $2 \mathrm{~b}$. Of the 6 branches from the third intercostal space, $4(66.7 \%)$ ran through area $3 \mathrm{a}$. Of the 6 branches from the fourth intercostal space, $3(50 \%)$ ran through areas $3 \mathrm{a}$ and $4 \mathrm{a}$.

The depth of ITA-perforating branches. At point S, the mean ( \pm standard deviation) distance from the skin to the branch and adipose thickness was $2.7 \pm 1.9 \mathrm{~mm}$ (range, 0-7.7) and 18.6 $\pm 9.4 \mathrm{~mm}$ (range, 3.7-40.8), respectively. The mean b/a ratio was $0.14 \pm 0.06$ (range, 0.0-0.24). A simple regression analysis showed a strong correlation between the distance from the skin to the branch and adipose thickness ( $r=0.737$, $\mathrm{P}<0.001$, Fig. 3). At the shallowest point, the distance from skin to the artery was $14 \%$ of adipose thickness. A simple regression analysis showed the relationship between the distances $\mathrm{S}$ and $\mathrm{N}$ ( $\mathrm{r}=0.454, \mathrm{P}=0.005$, Fig. 4). The distance from the sternum to the shallowest point was $57 \%$ of the distance to the nipple. Distance $\mathrm{S}$ did not differ significantly between branches originating in the second, third or fourth intercostal spaces (Scheffe's F test). 
At point $\mathrm{D}$, the mean distance from the skin to the branch and adipose thickness were $9.5 \pm 5.7 \mathrm{~mm}$ (range, 2.0-24.5) and $14.6 \pm 8.8 \mathrm{~mm}$ (range, 2.3-36.3), respectively. The mean b/a ratio was $0.69 \pm 0.20$ (range, $0.25-1$ ). As with point $S$, a simple regression analysis showed a strong correlation between the distance from the skin to the branch and thickness of adipose tissue ( $\mathrm{r}=0.814, \mathrm{P}<0.001$, Fig. 5). At the deepest point, the distance from the skin to the branch was $53 \%$ of adipose thickness. However, no correlations were noted between distances D and $\mathrm{N}$.

\section{Discussion}

This study of 38 patients who underwent MDCT angiography demonstrated locations of the origin and courses of ITAperforating branches. This artery typically originates in the second intercostal space and runs in a horizontal direction with a meandering course. Depth information was also obtained for the branches. For surgeons making skin and fat flaps with preserved perforating branches of the ITA, such distribution and depth information can prove very helpful, and individualized information for this artery can help to determine the flap shape.

Major arteries supplying the mammary gland are the lateral thoracic and thoracodorsal artery and, perforating branches of the ITA. The lateral thoracic and thoracodorsal artery flow from the outside. Breast reconstruction techniques considering these arteries have been reported. Hamdi et al (13) reported the use of a pedicled skin- and fat-only flap based on a perforating branch of the thoracodorsal and the intercostal artery and that technique had fewer complications compared to the muscle flap whose perforator was tiny or not-pulsating. Conversely, the arterial supply from the inside of the mammary gland only occurs via the ITA-perforating branches. However, reconstruction techniques using ITAperforating branches have yet to be described. This lack of a reported technique is likely due to the absence of detailed information on ITA-perforating branches.

Few studies of bifurcation areas for ITA branches have been reported (17-19). Rosson et al (17) noted that most perforating branches of the ITA diverge from the second intercostal space, followed by those from the third. The present results support these findings. As a premise for using ITA-perforating branches as recipient vessels for free flaps, Munhoz et al (19) described the location of the origin and caliber of the branches. These studies have covered only the original part of ITA-perforating branches. To the best of our knowledge, no reports have detailed the course, length or distribution of ITA-perforating branches using any imaging modality.

In this study, no perforating artery was detected in 2 cases. As no differences between these and the other cases were noted following the injection of contrast material, there was no reason to question the presence of perforating branches. However, as these cases did not undergo a DSA study, we could not confirm the presence of a perforating artery. The findings of this study are limited because a DSA study, which is the gold standard, was not conducted for any of the cases. Many reports in recent years have described MDCT angiography as an alternative method for the assessment of peripheral arteries (14-16). Therefore, the aim of this study was to consider the main perforating branches of the ITA, given that MDCT angiography can display these vessels as well as DSA.

DSA is a gold-standard examination and provides twodimensional data. The courses and distributions of ITAperforating branches may be able to be shown by DSA. However, one of the most important pieces of information when making a fat flap using ITA-perforating branches is arterial depth. In this study, a minimally invasive MDCT angiography demonstrated the curving course and depth information of ITA-perforating branches.

This study investigated ITA-perforating branches in breast cancer patients. A statistical analysis demonstrated that arterial depth at the shallowest and deepest points may be predicted from adipose thickness at those points. The results also show tendencies for intercostal origins and distributions of perforating branches. When making a fat flap involving ITA-perforating branches, the most important point of notice is arterial damage. Arterial depth information is thus crucial in such reconstructive surgery. The present findings should lead to innovations in immediate reconstructive techniques for surgical procedures following breast-conserving surgery. If a surgeon can make a pedicle flap after considering ITAperforating branch patterns in immediate breast reconstruction as a customized breast surgery, this technique can be expected to lead to a decrease in complications.

\section{Acknowledgements}

We wish to thank Takashi Hatakeyama, Kazuhiro Okino and Akiko Harada, radiology technologists at Kochi Medical School Hospital, for MDCT data acquisition.

\section{References}

1. Cody HS III: Current surgical management of breast cancer. Curr Opin Obstet Gynecol 14: 45-52, 2002.

2. Sakorafas GH: Breast cancer surgery - historical evolution, current status and future perspectives. Acta Oncol 40: 5-18, 2001 .

3. Asgeirsson KS, Rasheed T, McCulley SJ and Macmillan RD: Oncological and cosmetic outcomes of oncoplastic breast conserving surgery. Eur J Surg Oncol 31: 817-823, 2005.

4. Noguchi M, Taniya T, Miyazaki I and Saito Y: Immediate transposition of a latissimus dorsi muscle for correcting a postquadrantectomy breast deformity in Japanese patients. Int Surg 75: 166-170, 1990.

5. Banic A, Boeckx W, Greulich M, Guelickx P, Marchi A, Rigotti $\mathrm{G}$ and Tschopp H: Late results of breast reconstruction with free TRAM flaps: a prospective multicentric study. Plast Reconstr Surg 95: 1195-1204, 1995.

6. Park MC, Lee JH, Chung $\mathrm{J}$ and Lee SH: Use of internal mammary vessel perforator as a recipient vessel for free TRAM breast reconstruction. Ann Plast Surg 50: 132-137, 2003.

7. Tachi M and Yamada A: Choice of flaps for breast reconstruction. Int J Clin Oncol 10: 289-297, 2005.

8. Raja MA, Straker VF and Rainsbury RM: Extending the role of breast-conserving surgery by immediate volume replacement. Br J Surg 84: 101-105, 1997.

9. Kat CC, Darcy CM, O'Donoghue JM, Taylor AR and Regan PJ: The use of the latissimus dorsi musculocutaneous flap for immediate correction of the deformity resulting from breast conservation surgery. Br J Plast Surg 52: 99-103, 1999.

10. Dixon JM, Venizelos B and Chan P: Latissimus dorsi mini-flap: a technique for extending breast conservation. Breast 11: 58-65, 2002. 
11. Lin KY, Johns FR, Gibson J, Long M, Drake DB and Moore MM: An outcome study of breast reconstruction: presurgical identification of risk factors for complications. Ann Surg Oncol 8: 586-591, 2001

12. Birido N and Geraghty JG: Quality control in breast cancer surgery. Eur J Surg Oncol 31: 577-586, 2005.

13. Hamdi M, Van Landuyt K, Monstrey S and Blondeel P: Pedicled perforator flaps in breast reconstruction: a new concept. Br J Plast Surg 57: 531-539, 2004.

14. Kock MC, Adriaensen ME, Pattynama PM, van Sambeek MR, van Urk H, Stijnen T and Hunink MG: DSA versus multidetector row CT angiography in peripheral arterial disease: randomized controlled trial. Radiology 237: 727-737, 2005.

15. Schertler T, Wildermuth S, Alkadhi H, Kruppa M, Marincek B and Boehm T: Sixteen-detector row CT angiography for lower-leg arterial occlusive disease: analysis of section width. Radiology 23: 649-656, 2005.
16. Willmann JK, Baumert B, Schertler T, Wildermuth S, Pfammatter T, Verdun FR, Seifert B, Marincek B and Böhm T: Aortoiliac and lower extremity arteries assessed with 16-detector row CT angiography: prospective comparison with digital subtraction angiography. Radiology 236: 1083-1093, 2005.

17. Rosson GD, Holton LH, Silverman RP, Singh NK and Nahabedian MY: Internal mammary perforators: a cadaver study. J Reconstr Microsurg 21: 239-242, 2005.

18. Kalender V, Aydm H, Karabulut AB, Ozcan M and Amiraslanov A: Breast reconstruction with the internal mammary artery pedicled fasciocutaneous island flap: description of a new flap. Plast Reconstr Surg 106: 1494-1498, 2000.

19. Munhoz AM, Ishida LH, Montag E, Sturtz GP, Saito FL, Rodrigues L, Gemperli R and Ferreira MC: Perforator flap breast reconstruction using internal mammary perforator branches as a recipient site: an anatomical and clinical analysis. Plast Reconstr Surg 114: 62-68, 2004. 